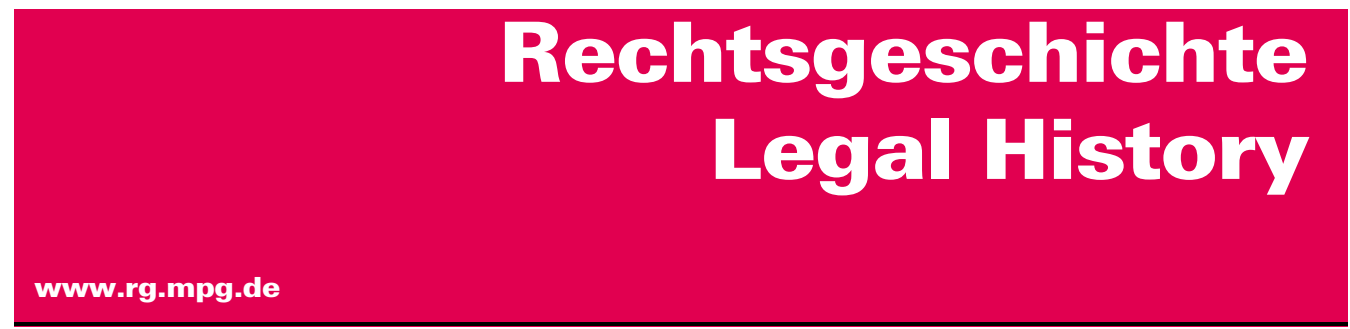

http://www.rg-rechtsgeschichte.de/rg28

Zitiervorschlag: Rechtsgeschichte - Legal History Rg 28 (2020)

$\operatorname{Rg} 282020$

$134-139$

http://dx.doi.org/10.12946/rg28/134-139

\title{
Carsten Fischer*
}

Andreas Thier **

\section{"Computing all their cost and trouble«- Finanzmärkte, Spekulation und Regulierung seit der Frühen Neuzeit: Diskurse, Narrative, Strategien. Eine Einleitung}

[»Computing all their cost and trouble« - Financial Markets, Speculation and Regulation since Early Modernity: Discourses, Narratives, Strategies. An Introduction]

* Universität zu Köln, Rechtswissenschaftliche Fakultät / Universität Trier, Fachbereich V - Rechtswissenschaft, carsten.fischer@uni-koeln.de

** University of Zurich, thier@access.uzh.ch 


\section{Carsten Fischer, Andreas Thier \\ "Computing all their cost and trouble« - Finanzmärkte, Spekulation und Regulierung seit der Frühen Neuzeit: Diskurse, Narrative, Strategien. Eine Einleitung}

Die Finanzmärkte, ihre Verflechtungen und die Volatilität ihrer Dynamiken sind identitätsbildende Merkmale unserer globalisierten Gegenwart. Dazu gehört auch die Erfahrung von gewaltigen Krisen wie etwa die Verwerfungen der Jahre 2007-2010. ${ }^{1}$ Sie haben regelmäßig Bemühungen ausgelöst - vor allem im Interesse des Anlegerschutzes, der Systemstabilität und damit der nationalen wie der globalen Wirtschaftsordnungen insgesamt -, dem Handeln der Marktakteure mit den Mitteln des Rechts Regeln zu setzen und insbesondere deren Befolgung durch die Begründung von Aufsichtsinstitutionen ${ }^{2}$ sicherzustellen. ${ }^{3}$ In dieser Hinsicht verweist das Recht der Finanzmärkte als Ergebnis eines spannungsvollen Wechselspiels von »Crises and Opportunities « ${ }^{4}$ auf die grundlegende Beziehung von Risikokulturen, Krisenerfahrungen und die Entwicklungsdynamiken von Recht und Rechtswissen.

Diese Verflechtungen sind durchaus wahrgenommen, aber doch vergleichsweise selten ausgeleuchtet worden. So wurde die Geschichte des Kapitalmarktrechts und des Anlegerschutzes seit dem späten 19. Jahrhundert - jedenfalls im europäischen Zusammenhang - immer wieder in den Blick genommen. Auch die »Krise« als historisches
Phänomen hat seit der klassischen Studie Reinhart Kosellecks ${ }^{5}$ immer häufiger den Anknüpfungspunkt geschichtswissenschaftlicher Analysen gebildet. ${ }^{6}$ Aber auch wenn sich die Rechtsgeschichte der Finanzmärkte zumindest in der Zeit des 19. und 20. Jahrhunderts in gewisser Hinsicht als Rechtsgeschichte von Krisenreaktionen erfassen ließe, ${ }^{7}$ so scheinen doch systematische Untersuchungen über diese Zusammenhänge eher selten zu sein. Das gilt auch mit Blick auf das »Risiko«: Es hat als perspektivbildende Kategorie der historischen Analyse in jüngerer Zeit zunehmend das Interesse der Forschung gefunden ${ }^{\mathbf{8}}$ und steht nicht zuletzt auch im Zusammenhang mit der Entstehung von Wahrscheinlichkeitsrechnung und Kalkulation. ${ }^{9}$ Umgekehrt ist die Frage nach dem Umgang von Recht mit Risiken seit langem und aus unterschiedlichen Perspektiven auch ein Thema der rechtswissenschaftlichen Debatte. ${ }^{\mathbf{1 0}}$ Aber die Frage nach der Geschichtlichkeit rechtlicher Risikokulturen ist, soweit ersichtlich, bislang eher selten gestellt worden, ${ }^{\mathbf{1 1}}$ auch wenn sich vermuten lässt, dass hier ein ertragreiches Feld nicht nur, aber insbesondere der rechtshistorischen Forschung liegt. ${ }^{\mathbf{1 2}}$ Mit Rechtsfiguren und Semantiken wie »Zeitgeschäft «, ${ }^{\mathbf{1 3}}$ »Futures ${ }^{\mathbf{1 4}}$ oder $»$ Termin-
1 Dazu etwa die Darstellung bei LYBeCK (2011).

2 Avgouleas (2012).

3 Umfassend Moloney et al. (Hg.) (2015).

4 CAssis (2011).

5 Koselleck (1973/2010).

6 Aus jüngerer Zeit etwa die Beiträge in David et al. (Hg.) (2012).

7 Dazu Nobel (2019) für die Gegenwart, aus historischer Perspektive Contratto (2013) und Thier (2017a), Rdnrn. 3, 33 m. w. N.; zurückhaltend gegenüber der Bedeutung von Krisen als Katalysator wirtschaftsrechtlichen Wandels dagegen SCHMOECKel (2015).

8 S. etwa die Beiträge bei Christians / Mein (Hg.) (2019), Contzen et al. (Hg.) (2018) oder nunmehr die grundlegenden Beiträge zu einer Geschichte des Risikos im spätmittelalterlichen Europa von SCheller (2017) und (2018).

9 Frankin (2001); Hacking (1990) und (2006).

10 Grundlegend: LuhmanN (1991); als Überblick Thier (2017) 845-849, näher NoBEL (2010).
11 S. dazu die Überblicke zur Forschung bei Contzen / Huff et al. (2018) sowie bei ItZen / MüLler (2016).

12 Als skizzenhafter Überblick siehe Thier (2017) m. w. N.; grundlegend NeHLSEN-von Stryk (1989).

13 Cohn (1867), Moser (1875) und zu ihm Schmidt (2009).

14 Dazu aus deutscher Perspektive etwa Menninger (1994), aus Sicht der Globalgeschichte ENGEL (2015), passim. 
geschäft ${ }^{15}$ wird nicht allein ein Ausschnitt der komplexen Beziehungsgeschichte von Recht und Temporalität ${ }^{\mathbf{1 6}}$ sichtbar. Insbesondere das breite Spektrum von Bewertungen der "Spekulation « ${ }^{\mathbf{1 7}}$ und ihrer Folgen belegt eine auch historisch schwankende Vielfalt unterschiedlicher Wahrnehmungen der Dynamiken von Finanzmärkten und ihrer Umbrüche. Eine regelrechte Konstante bilden dabei die Darstellungen spekulativer Blasenbildungen als Ergebnis menschlicher Gier und Bösartigkeit. In Jonathan Swifts Gedicht »The Bubble« von 1720, entstanden in der Zeit einer massiven Spekulationskrise, ${ }^{\mathbf{1 8}}$ heißt es etwa: »The nation then too late will find, Computing all their cost and trouble, Directors' promises but wind, South-Sea, at best, a mighty bubble. " ${ }^{19}$ Auf einer ähnlichen Ebene bewegte sich die Subsumtion von spekulativen Blasenbildungen als »Manias«, die in einer popularisierenden Darstellung von Charles Mackay als Ausdruck von Extraordinary Popular Delusions and the Madness of Crowds $(1841)^{\mathbf{2 0}}$ gedeutet wurden. Allerdings sind Krisen und Umbrüche der frühneuzeitlichen und modernen $\mathrm{Fi}^{-}$ nanzmärkte auch zum Ausgangspunkt für die Analyse von Preisbildungsprozessen als »Fundamentals of Early Manias « geworden, ${ }^{21}$ sie haben Untersuchungen über Anlagestrategien im Zentrum von Spekulationsblasen angeregt ${ }^{22}$ und $\mathrm{zu}$ Diskussionen über den Zusammenhang von Innovation und Blasenbildung an den Finanzmärkten geführt. ${ }^{23}$ Jenseits davon ist auch versucht worden, die Verwerfungen der Finanzmärkte in ihrer Entstehung und in ihren Ursachen im historischen
Längsschnitt zu vergleichen, ${ }^{\mathbf{2 4}}$ um auf dieser Grundlage nicht zuletzt Strategien zur Bewältigung künftiger Krisen möglich zu machen. Das schließt insbesondere die Frage ein, ob und wie Finanzmärkte mit den Instrumenten und Institutionen hoheitlicher Herrschaft gebändigt werden können, wie das im Ausgangspunkt etwa mit rechtlichen Normen über das Geldrecht ${ }^{25}$ und später durch staatliche Zentralbanken ${ }^{26}$ sowie die Regulierung des Banken- und Finanzmarktes ${ }^{27}$ vor allem auch im Interesse des Anlegerschutzes ${ }^{28}$ versucht wurde. In gewissem Maße verweisen solche Entwicklungen auf die Geschichte des Spannungsfeldes zwischen der Entstehung des »Vorsorgestaates ${ }^{29}$ mit der staatlichen Letztverantwortung über Risikoentscheidungen ${ }^{\mathbf{3 0}}$ einerseits und der Verbindlichkeitsgarantie privatautonomen Risikohandelns andererseits.

Diese Diskurse, Narrative und Strategien sind in unterschiedlicher Weise in den nachfolgenden sieben Beiträgen untersucht worden. ${ }^{31}$ Michael North und Andreas Thier skizzieren grundlegende Strukturen des Geldmarktes und seiner Regulierung durch die hoheitliche Gewalt in der Zeit seit dem ausgehenden Mittelalter. Zwar zählte die Kontrolle über Währungen, Geldwert und Finanzmärkte seit dem späten Mittelalter stets zum Anspruch hoheitlicher Gewalt; aber die Durchsetzung solcher Ansprüche ist, so zeigt sich im Blick auf die Vielzahl von krisenhaften Umbrüchen etwa in Gestalt von Währungskrisen, Bankenzusammenbrüchen oder Spekulationsblasen, zu keinem Zeitpunkt sicher. Gerade im Bereich der Finanzmärkte stößt die
15 Aus historischer Perspektive Wolter (1991) mit Blick auf die deutsche Regelung.

16 Dazu die Überlegungen bei THIER (2017b).

17 Grundlegend dazu die Beiträge von ENGEL (2013) und (2016); s. a. StÄHeli (2007) sowie den Beitrag von Engel und Flume in diesem Fokus.

18 Dazu der Beitrag von North und Thier in diesem Fokus.

19 SwIFt (1720/1958) 259. Zum Kontext und zur Deutung des Gedichts s. Moore (2010) 59-89; Rogers (1988).

20 MackAY (1841), Bd. 1 mit dem bezeichnenden Untertitel »Economic Bubbles«.

21 Garber (2000); s. a. Bilginsoy (2015).
22 Temin/Voth (2004) und (2013), Dale et al. (2005).

23 S. etwa Neal (1991) 90.

24 Klassisch ReInHart/Rogoff (2009).

25 Dazu die Beiträge in Fox/ERnST (Hg.) (2016) sowie nunmehr bei Battilossi et al. (Hg.) (2020), v. a. 107-219.

26 Klassisch Goodhart (1988), aus jüngster Zeit Ugolini (2017) und jetzt die Analysen bei BattiLossi et al. (Hg.) (2020) 833-979.

27 Dazu etwa die Studien in Cassis et al. (Hg.) (2016) sowie ENGEL (2012) und Fleckner/Hopt (2013).

28 Allgemein Hopt (1975), Sethe (2005), Thier (2013).

29 Ewald (1993).

30 Di Fabio (1994/2019).
31 Die Texte gehen zurück auf Beiträge zum Workshop »Computing all their cost and trouble - Finanzmärkte, Spekulation und Regulierung in der Frühen Neuzeit und in der Moderne. Diskurse, Narrative, Strategien«, der im Oktober 2015 von Carsten Fischer und Andreas Thier in Zürich veranstaltet wurde. Jan Thiessens Beitrag »Vom director zum Führer und zurück - historisch-vergleichende Anmerkungen zur Organisationsverfassung von stock corporation und Aktiengesellschaft « konnte leider wegen anderweitiger Verpflichtungen des Beitragenden nicht für den Druck überarbeitet werden. Einige der Überlegungen finden sich jedoch in Thiessen (2013), insbes. ab 235. 
hoheitliche Herrschaft an die Grenzen ihrer Wirksamkeit.

Wirtschaftlicher Erfolg und Ansehen gingen auch und erst recht in der Frühen Neuzeit nicht Hand in Hand, im Gegenteil: Der Adel etwa war als gesellschaftliche Funktionselite geradezu darauf angewiesen, seine Stellung als nicht an wirtschaftliche Erfolge gebunden zu demonstrieren. ${ }^{32}$ Gleichwohl veränderte wirtschaftliche Potenz auch in der Frühen Neuzeit die eigenen Möglichkeiten nicht nur des Adels natürlich deutlich. Daher spürt Christian Kullick einer gesellschaftlichen Verunsicherung nach, wenn er exemplarisch Lotterien im Frankfurt am Main der zweiten Hälfte des 18. Jahrhunderts untersucht. Die Aussicht auf plötzlichen Reichtum stellte die Frage nach der Erwartungssicherheit der jeweiligen Stellung im gesellschaftlich-ständischen Gefüge. Damit war der städtische Rat zunächst als Garant der sozialen Ordnung gefordert - auf Grund der mit dem Lotteriebetrieb verbundenen Einnahmemöglichkeiten, aber auch als weiser Fiskal, dem in dieser Rolle daran gelegen sein musste, Frankfurt als regional attraktiven Marktplatz für Lotterien zu etablieren. Die Analyse des Verlaufes des »Lotteriefiebers" in Frankfurt ist eine Mikrostudie zu Regulierungsstrategien innerhalb der komplexen frühneuzeitlichen Interessenmatrix. In der Einbeziehung der Durchsetzbarkeit und tatsächlichen Durchsetzung erklärt Christian Kullick gleichzeitig Strategieanpassungen in der Lotterieregulierung.

Mit dem Beitrag von Andreas Engel und Johannes W. Flume wandert der Blick gut ein Jahrhundert weiter, die Fragestellung lässt sich jedoch mit der Untersuchung des Frankfurter Lotteriefiebers verknüpfen: Wer ist auf einem komplexen wirtschaftlichen Parkett hochriskanter Geschäfte weshalb schutzbedürftig - und wer hat für diesen Schutz zu sorgen? In der Frage nach der Schutzbedürttigkeit von weniger professionellen Anlegern - den "Lämmern" des Wirtschaftslebens - scheint hier erneut ein Hauptmovens von Regulierungstätigkeit auf. Die Debatte über Schutzmechanismen reichte im deutschen Kaiserreich Ende des 19. Jahrhunderts von staatlicher Regulierung bis zu Selbstverpflichtungen der am Börsengeschehen Beteiligten, (vielfach nur behauptete) professionelle, aber auch charakterliche Standards zu gewährleisten. Auch wenn vornehmlich das Schicksal der »Läm- mer« diskutiert wurde - der Schutz einzelner Anleger konnte auch zum Schutz der Wirtschaftsordnung insgesamt fortgedacht werden.

Der Beitrag Dieter Zieglers richtet den Blick auf Regulierungsmechanismen des englischen Finanzmarktgeschehens, deren Schwerpunkt jenseits rechtlicher Ordnungsgefüge lag: Innerhalb eines durch Gesetzgebung, insbesondere die Peelsche Bankakte, wie auch durch Selbstregulierung rechtlich eingehegten Systems erscheinen das an Autosuggestion grenzende Vertrauen auf (angenommene) wirtschaftliche Grundregeln des Finanzmarktes und ein an ihnen ausgerichtetes Verhalten als mindestens ebenso bedeutsam für die Vermeidung oder Abschwächung von Krisen wie etwa die Steuerung mit legislativen Mitteln. Unerlässlich für das Verständnis auch der Krisentauglichkeit solcher Erwartungen sei es allerdings, so Dieter Ziegler, die Politik der Bank of England nicht nur als Auftreten einer Bank der Banken (lender of last resort), sondern als diejenige einer im handfesten Wettbewerb mit anderen Finanzinstituten stehenden Geschäftsbank zu betrachten.

Louis Pablow spürt mit der Gegenüberstellung der Regulierung von Aktiennotenbanken (»Zettelbanken«) und Aktienkreditbanken der Finanzmarkt- und Geldpolitik des deutschen Kaiserreichs nach. Geht man vom weit verbreiteten Bild des zusehends vorsorgenden Interventionsstaates aus, ist das Ergebnis dieser Studie fast schon ernüchternd: Eine komplexere langfristige Regulierungsstrategie des Reiches wird mit Blick auf den Finanzmarkt nicht erkennbar. Vielmehr zeigt sich im Abgleich der unterschiedlichen Regulierung von Zettel- und Aktienkreditbanken das Ziel einer Lenkung vorrangig des Notenbankwesens und somit der Geldpolitik. Das Reich agierte hier gerade nicht als Interventionsstaat. In dieser vornehmlich geldpolitischen Ausrichtung der Bankenregulierung steht das Kaiserreich damit trotz der verfassungsrechtlichen Besonderheit, stets die Interessen und Kompetenzen der Länder mit berücksichtigen zu müssen, dem von Dieter Ziegler herausgearbeiteten englischen Regulierungsmodell nicht fern.

Ein Beispiel komplexer Selbstregulierung untersucht Peter Collin am Konkurrenzverhältnis zwischen Sparkassen, Banken und Kreditgenossenschaften im Deutschland des frühen 20. Jahrhun-

32 Hierzu jüngst Solterbeck (2018). 
derts. Gerade die dieser Zeit eigenen dramatischen Änderungen der wirtschaftlichen Rahmenbedingungen sorgten für die Verschiebung von Geschäftsfeldern und eine sich verschärfende Konkurrenzlage im Bankgeschäft. In dieser Situation füllten die Wettbewerber den nur sehr weit und insbesondere für Sparkassen gesetzten Rahmen staatlicher Regeln auch mit Blick auf möglicherweise erwartete weitere Regulierung durch ein komplexes Geflecht von Abkommen untereinander. Diese insbesondere zwischen 1918 und 1932 eingegangenen Vereinbarungen schufen so ein differenziertes System regulierter Selbstregulierung, das unter den Beteiligten nicht nur Normen setzte und konkretisierte, sondern auch Regeln und Institutionen zur Normdurchsetzung etablierte - gewissermaßen eine Krisenbewältigung durch konkurrierende Kooperation. An Louis Pablows Befunde anknüpfend zeigt sich etwa in der gesetzgeberischen Zurückhaltung eine regulatorische Konstante des deutschen Staates zwischen »Gründerkrise und NS-Zeit.

Der diesen Fokus schließende Beitrag steht als literaturwissenschaftliche Deutung neben den bis- her genannten rechts- und wirtschaftshistorischen Texten, überwölbt sie aber gleichsam: Jurij Murašov analysiert ausgewählte literarische Spiegelungen hochkomplexer moderner wirtschaftlicher Vorgänge. In seiner Interpretation moderner Narrative des Wirtschaftsgeschehens hebt er auch ihre Übersetzungs- wie Konstruktionsleistung hervor: Wo Ökonomie, etwa auf Grund der Geschwindigkeit ihrer Transaktionen oder der Entkopplung der Finanzmärkte von einer produktionsbasierten Wirtschaft, sich aus bisherigen Erklärungen und Beschreibungen löst und - zumindest für die meisten Beobachter, aber (wie zu vermuten steht) auch für viele Akteure - in wesentlichen Teilen nicht mehr nachvollziehbar ist, kommt der Beschreibung dieser Vorgänge durch Literatur eine besondere Bedeutung zu. Vielleicht scheint hier eine Stärke von Narrativen auf: Gerade der Umstand, dass sie die Fachdiskurse zwar berühren, sich ihren Regeln aber nicht verpflichtet fühlen, erlaubt es ihnen, eine neue Erzählbarkeit zu schaffen.

\section{Bibliographie}

- Avgouleas, Emilios (2012), Governance of Global Financial Markets. The Law, the Economics, the Politics, Cambridge

- Battilossi, Stefano et al. (Hg.) (2020), Handbook of the History of Money and Currency, Singapur

- Bilginsoy, Cihan (2015), A History of Financial Crises. Dreams and Follies of Expectations, London

- Cassis, Youssef (2011), Crises and Opportunities. The Shaping of Modern Finance, Oxford

- Cassis, Youssef et al. (Hg.) (2016), The Oxford Handbook of Banking and Financial History, Oxford

- Christians, Heiko, Georg Mein (Hg.) (2019), Gefahr oder Risiko. Zur Geschichte von Kalkül und Einbildungskraft, Paderborn

- Cohn, Gustav (1867), Zeitgeschäfte und Differenzgeschäfte. Volkswirthschaftliche Studie, Jena

- Contratto, Franca (2013), Das Anlegerleitbild im Wandel der Zeiten, in: Sethe et al. (Hg.) 47-83

- Contzen, Eva von et al. (Hg.) (2018), Risikogesellschaften. Literatur- und geschichtswissenschaftliche Perspektiven, Bielefeld

- Contzen, Eva von, Tobias Huff et al. (2018), Risikogesellschaften: Eine Einführung. Reden über Risiko jenseits der Disziplinengrenzen, in: Contzen et al. (Hg.) 7-32

- Dale, Richard J., Johnnie E. V. Johnson et al. (2005), Financial markets can go mad: evidence of irrational behaviour during the South Sea Bubble, in: The Economic History Review 58,2, 233-271

- David, Thomas et al. (Hg.) (2012), Krisen - Crises. Ursachen, Deutungen und Folgen. Causes, interpretations et consequences, Zürich

- Di Fabio, Udo (1994/2019), Risikoentscheidungen im Rechtsstaat. Zum Wandel der Dogmatik im öffentlichen Recht, insbesondere am Beispiel der Arzneimittelüberwachung, Tübingen

- Engel, Alexander (2012), Die Regulierung des Börsenterminhandels im Kaiserreich, in: Bankhistorisches Archiv, supplement 48, 27-39

- Engel, Alexander (2013), Vom verdorbenen Spieler zum verdienstvollen Spekulanten. Ökonomisches Denken über Börsenspekulation im 19. Jahrhundert, in: Jahrbuch für Wirtschaftsgeschichte/Economic History Yearbook 54,2, 49-70, https:// doi.org/10.1524/jbwg.2013.54.2.49

- Engel, Alexander (2015), Buying time: futures trading and telegraphy in nineteenth-century global commodity markets, in: Journal of Global History 10,2, 284-306, https://doi.org/10.1017/S1740022815000078

- Engel, Alexander (2016), "Ist nämlich der ganze Spekulationsverkehr erst einmal in einen krankhaft erregten Zustand hineingerathen ...«. Pathologien der Börse im späten 19. Jahrhundert, in: Jahrbuch für Wirtschaftsgeschichte/Economic History Yearbook 57,2, 333-365, https://doi.org/10.1515/jbwg-2016-0015 
- Ewald, François (1993), Der Vorsorgestaat, übersetzt v. Kocyba, Hermann, Wolfram Bayer, Frankfurt a. M. (Französisches Original: L'Etat providence, Paris 1986)

- Fleckner, Andreas M., Klaus J. Hopt (2013), Stock Exchange Law: Concept, History, Challenges, in: Virginia Law \& Business Review 7,3, 513-559, http://dx.doi.org/10.2139/ssrn.2068574

- Fox, David, Wolfgang Ernst (Hg.) (2016), Money in the Western Legal Tradition: Middle Ages to Bretton Woods, Oxford

- Franklin, James (2001), The Science of Conjecture. Evidence and Probability before Pascal, Baltimore (Neudruck 2015)

- Garber, Peter M. (2000), Famous First Bubbles. The Fundamentals of Early Manias, Cambridge/MA

- Goodhart, Charles (1988), The Evolution of Central Banks, Cambridge

- Hacking, IAn (1990), The Taming of Chance, Cambridge

- Hacking, Ian (2006), The Emergence of Probability. A Philosophical Study of Early Ideas about Probability, Induction and Statistical Inference, 2. Aufl., Cambridge

- Hopt, Klaus J. (1975), Der Kapitalanlegerschutz im Recht der Banken. Gesellschafts-, bank- und börsenrechtliche Anforderungen an das Beratungs- und Verwaltungsverhalten der Kreditinstitute, Tübingen

- Itzen, Peter, Simone M. Müller (2016), Risk as a Category of Analysis for a Social History of the Twentieth Century. An Introduction, in: Historical Social Research 41,1, 7-29

- Koselleck, Reinhart (1973/2010), Kritik und Krise. Eine Studie zur Pathogenese der bürgerlichen Welt, 11. Aufl., Frankfurt a. M.

- Luhmann, Niklas (1991), Soziologie des Risikos, Berlin

- Lybeck, Johan A. (2011), A Global History of the Financial Crash of 2007-2010, London

- Mackay, Charles (1841), Extraordinary Popular Delusions and the Madness of Crowds, London

- Menninger, Jutta (1994), Börsen- und zivilrechtlicher Charakter von Financial Futures, in: Wertpapier-Mitteilungen, 970-974

- Moloney, Niamh et al. (Hg.) (2015), The Oxford Handbook of Financial Regulation, Oxford

- Moore, Sean D. (2010), Swift, the Book, and the Irish Financial Revolution. Satire and Sovereignty in Colonial Ireland, Baltimore, https://muse.jhu.edu/book/475

- Moser, James (1875), Die Lehre von den Zeitgeschäften und deren Combinationen, Berlin

- Neal, Larry (1991), The Rise of Financial Capitalism. International Capital Markets in the Age of Reason, Cambridge

- Nehlsen-von Stryk, Karin (1989), Kalkül und Hasard in der spätmittelalterlichen Seeversicherungspraxis, in: Rechtshistorisches Journal 8, 195-208

- Nobel, Peter (2010), Der Risikobegriff und rechtliche Risiken, in: Zeitschrift für schweizerisches Recht 129,I, 3-27

- Nobel, Peter (2019), Schweizerisches Finanzmarktrecht, 4. Aufl., Bern

- Reinhart, Carmen M., Kenneth Rogoff (2009), This Time is Different. Eight Centuries of Financial Folly, Princeton

- Rogers, Pat (1988), Plunging in the Southern Waves: Swift's Poem on the Bubble, in: The Yearbook of English Studies 18, $41-50$

- Scheller, Benjamin (2017), Die Geburt des Risikos. Kontingenz und kaufmännische Praxis im mediterranen Seehandel des Hoch- und Spätmittelalters, in: Historische Zeitschrift 304, 305-331

- Scheller, Benjamin (2018), (Un-)sichere Häfen. Häfen als Hotspot maritimer Risiken und Risikokommunikation im Mittelmeerraum des 15. Jahrhunderts, in: Historische Anthropologie. Kultur - Gesellschaft - Alltag 26,1, 43-65

- Schmidt, Hartmut (2009), Retrospective Book Review on James Moser: »Die Lehre von den Zeitgeschäften und deren Combinationen« (1875), in: Hafner, Wolfgang, Heinz Zimmermann (Hg.),Vinzenz Bronzin’s Option Pricing Models, Berlin, $467-470$

- Schmoeckel, Mathias (2015), »Gründerkrise« und »Grosse Depression«. Zur notwendigen Revision einer Geschichtsdeutung, in: Zeitschrift für Rechtsgeschichte (Germanistische Abteilung) 132, 251-322

- Sethe, Rolf (2005), Anlegerschutz im Recht der Vermögensverwaltung, Köln

- Sethe, Rolf et al. (Hg.) (2013), Anlegerschutz im Finanzmarktrecht kontrovers diskutiert, Zürich

- Solterbeck, Sven (2018), Blaues Blut und rote Zahlen. Westfälischer Adel im Konkurs 1700-1815, Münster

- Stäheli, Urs (2007), Spektakuläre Spekulation - Das Populäre der Ökonomie, Frankfurt a. M.

- Swift, Jonathan (1720/1958), The Bubble, in: Williams, Harold (Hg.), The Poems of Jonathan Swift, Bd. 1, 2. Aufl., Oxford, 248-259 (Oxford Scholarly Editions Online [2014], https://www.oxfordscholarlyeditions.com/view/10.1093/actrade/978019 9670352.book.1/actrade-9780199670352-book-1)

- Temin, Peter, Hans-Joachim Voth (2004), Riding the South Sea Bubble, in: The American Economic Review 94,5, 1654-1668, www.jstor.org/stable/3592838

- Temin, Peter, Hans-Joachim Voth (2013), Prometheus Shackled. Goldsmith Banks and England's Financial Revolution after 1700, Oxford

- Thier, Andreas (2013), Beobachtungen zur Geschichte des Anlageschutzes, in: Sethe et al. (Hg.) 26-45

- Thier, Andreas (2017), »Mala futura«, »securitas« und »Spekulation«: Rechtskulturen des Risikos im historischen Wandel, in: Weber, Rolf H. et al. (Hg.), Aktuelle Herausforderungen des Gesellschafts- und Finanzmarktrechts. Festschrift für Hans Caspar von der Crone zum 60. Geburtstag, Zürich, 845-862

- Thier, Andreas (2017a), Vor Art. 1: Zur historischen Verortung des FinfraG, in: Sethe, Rolf et al. (Hg.), Kommentar zum Finanzmarktinfrastrukturgesetz (FinfraG), Zürich, 30-49

- Thier, Andreas (2017b), Time, Law, and Legal History - Some Observations and Considerations, in: Rechtsgeschichte - Legal History 25, 20-44, http://dx.doi.org/10.12946/rg25/020-044

- Thiessen, Jan (2013), Wirtschaftsrecht und Wirtschaftsrechtler im Schatten der NS-Vergangenheit, in: Görtemaker, Manfred, Christoph Safferling (Hg.), Die Rosenburg. Das Bundesministerium der Justiz und die NS-Vergangenheit. Eine Bestandsaufnahme, Göttingen, 204-295 
- Ugolini, Stefano (2017), The Evolution of Central Banking: Theory and History, London

- Wolter, Udo (1991), Termingeschäftsfähigkeit kraft Information. Eine rechtshistorische, rechtsdogmatische und rechtspolitische Studie über die stillschweigende Entfunktionalisierung des $\$ 764$ BGB durch die Börsengesetz-Novelle 1989, Paderborn 